\title{
Size Effect in Fiber or Bar Pullout with INTERFACE Softening SLIP
}

\author{
By Zdeněk P. Bažant,' Fellow, ASCE, and Rodrigue Desmorat ${ }^{2}$
}

\begin{abstract}
The paper analyzes the size effect, which is an inevitable consequence of softening in the relation of interface shear stress and slip displacement between a fiber or reinforcing bar and the surrounding matrix. The problem is simplified as one-dimensional. Closed-form solutions of pull-pull and push-pull failures are obtained for a linear softening stress-slip law with residual strength, and for an exponential law without residual strength. The postpeak softening is shown to lead to localization of slip and interface shear fracture with a process zone of finite length. This zone propagates along the interface during the loading process, causing the distribution of interface shear stress to become strongly nonuniform. The larger the bar or fiber size, the stronger the nonuniformity. The size effect in geometrically similar pullout tests of different sizes is found to represent a smooth transition between two simple asymptotic cases: (1) The case of no size effect, which occurs for very small sizes and is characteristic of plastic failure; and (2) the case of a size effect of the same type as in linear elastic fracture mechanics, in which the difference of the pullout stress from its residual value is proportional to the inverse square root of the fiber or bar diameter. An analytical expression for the transitional size effect is obtained. This expression is found to approximately agree with the generalized form of the size effect law proposed by Bažant. The shape of the size effect curve is shown to be related to the shape of the softening stress-slip law for the interface. Finally, it is shown how measurements of the size effect can be used for identifying the interface properties, and a numerical example is given.
\end{abstract}

\section{INTRODUCTION}

The shear stress in the interface between fibers and matrix in composites or between steel bars and concrete is related to the slip displacement in the interface. This relation is known to exhibit a postpeak softening. When softening occurs, analysis of the failure load according to plasticity becomes invalid. One must take into account localization of softening damage along the interface and consider fracture mechanics aspects of the problem. By analogy with studies of strain-softening damage, one must also expect a size effect on the nominal strength of geometrically similar structures of different sizes, which represents the most important practical consequence of the localization of softening damage. The objective of this paper is to analyze this size effect.

The problem of pullout of fibers or bars from the surrounding matrix has received considerable attention in recent years and many important results have been achieved; see, e.g., Lawrence (1972); Freund (1992); Fuller et al. (1990); Gao et al. (1988); Leung and Li (1990a, b); Li et al. (1991); Shah and Ouyang (1991); Stang et al. (1990); Steif and Hoysan (1986); Wang et al. (1988); Beaumond and Aleszka (1978); Bowling and Groves (1979); and Gray (1984a, 1984b). An excellent review of the pullout test analysis has recently been presented by Shah and Ouyang (1991). Further

'Walter P. Murphy Prof. of Civ. Engrg., Northwestern Univ., Evanston, IL 60208.

${ }^{2}$ Visiting Res. Asst., Northwestern Univ., Evanston, IL.

Note. Discussion open until February 1, 1995. To extend the closing date one month, a written request must be filed with the ASCE Manager of Journals. The manuscript for this paper was submitted for review and possible publication on April 28, 1993. This paper is part of the Journal of Engineering Mechanics, Vol. 120, No. 9. September, 1994. CASCE, ISSN 0733-9399/94/0009-1945/\$2.00 + \$.25 per page. Paper No. 6017. 
light on the interface slip has been shed by studies of slip at interfaces of other types, including relative slip of rough crack surfaces [e.g., Bažant and Gambarova (1980), Divakar et al. (1987), and Feenstra et al. (1991)].

Most interface models consider the shear stress at the interface to be a function of the slip displacement [e.g., Bažant and Gambarova (1980) or Divakar et al. (1987)]. To make analytical solutions feasible, many previous authors have simplified the complex three-dimensional behavior at interface as one-dimensional [e.g., Gao et al. (1988) or Freund (1992)]. In the onedimensional solution, the influence of the normal pressure across the interface can be taken into account as long as this pressure is known. But if this pressure is unknown, a more general solution that takes into account

(a)

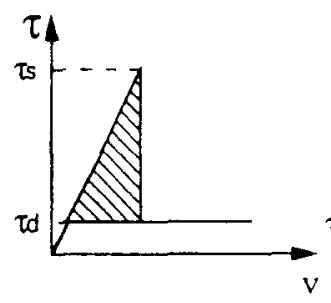

(b)

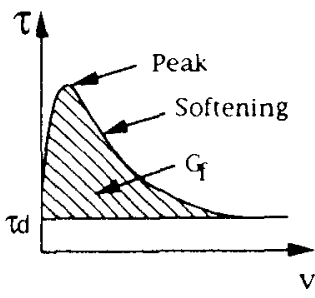

(c)

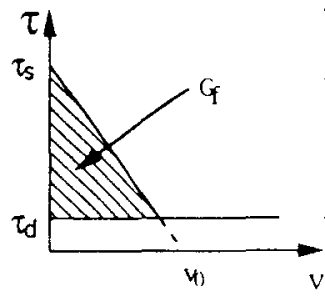

(d)

FIG. 1. Various Assumptions about Interface Properties Characterized in Terms of Interface Shear Stress and Relative Displacement

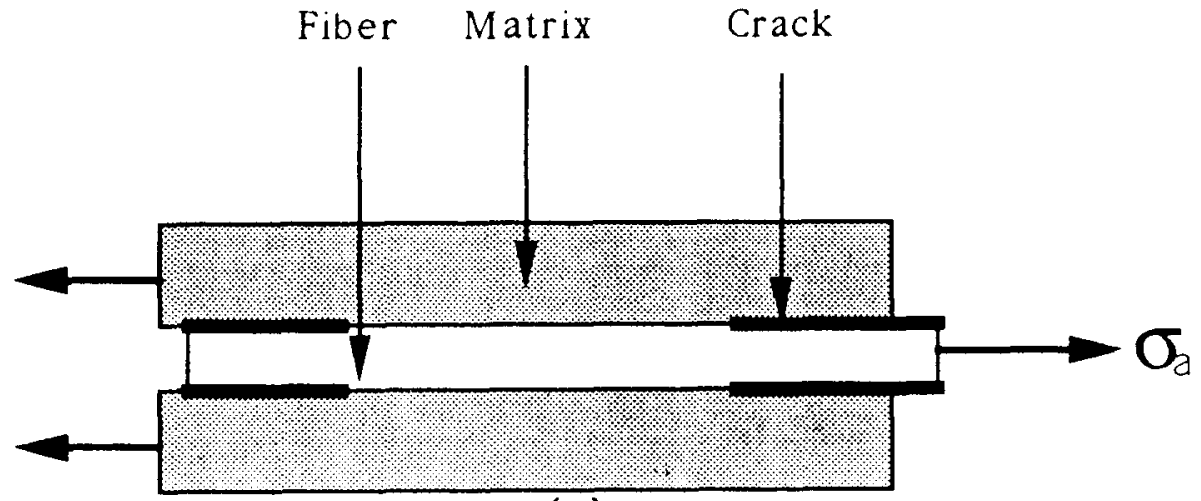

(a)

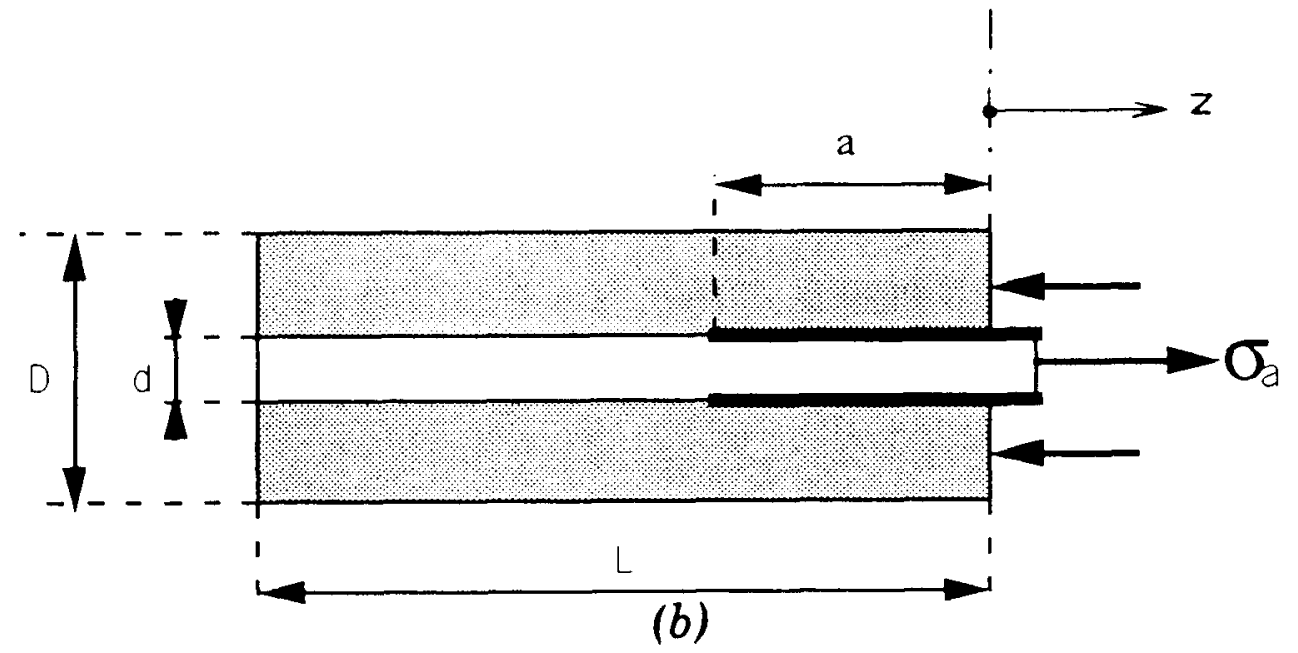

FIG. 2. Geometry of Fiber or Bar Pullout Tests: (a) Pull-Pull; and (b) Pull-Push 
the interface dilatancy, i.e., the normal relative displacement across the crack, is required. In the simplified one-dimensional analysis, the interface dilatancy can be approximately taken into account by adjusting the values of the parameters in the functional relationship $\tau(v)$ linking the interface shear stress $\tau$ to the relative slip displacement $v$; see, e.g., Lawrence et al. (1972), Bowling and Groves (1979), and Hutchinson and Jensen (1990). Stang et al. (1990) considered the stress-slip relation to consist of an elastic part followed by a sudden stress drop and a residual constant friction [Fig. $1(a)$ ]. However, it is no doubt more realistic to consider a gradual softening as shown in Fig. 1(b) and $(c)$ (for a sufficiently large fiber size, the sudden stress drop with an increased strength limit but the same area under the curve giving the fracture energy must nevertheless give approximately equivalent results). As for the rising initial linear stress-displacement relation shown in Fig. 2(a), it cannot be an interface property but must refer to the deformation in the layers of the matrix adjacent to the interface. For this reason, we will omit the rising linear part. As for the postpeak softening, we will consider it to be linear [Fig. 1(c)], in order to make a simple analytical solution feasible, although the real behavior is no doubt a smooth curve.

The size effect in the problem of fiber or bar pullout has apparently not yet been studied theoretically. However, its existence has already been demonstrated experimentally for the case of bar pullout from concrete (Bažant and Sener 1988). In this paper, we will focus on the analysis of the size effect, considering a situation with a two-way debonding similar to that of Leung and Li (1990). We will deduce closed-form analytical formulas for the size effect, consider the asymptotic cases, and finally show how knowledge of the size effect can be exploited for determining the interfacial material properties solely from measurements of the maximum pullout forces.

Because we will simplify the problem as one-dimensional, we will be unable to make a distinction between fibers in composites and reinforcing bars in concrete, except in terms of the effective values of material parameters (such as the bond strength or the residual bond stress). Fibers and bars differ in fracture patterns, dilatancy, and pressure sensitivity. But these phenomena can be specifically described only in a three-dimensional analysis.

\section{IDEALIZATION OF PROBLEM AND ASSUMPTIONS}

For the sake of simplicity, our analysis will be one-dimensional. A cylindrical fiber or bar of diameter $d$ is assumed to be embedded in an outer cylinder of diameter $D$ representing the matrix of a composite material (Fig. 2 ). The cross sections of the fiber or bar and of the outer cylinder are assumed to remain planar, but relative slip at the interface is possible. The stresses within the fiber as well as the matrix are uniform in each cross section. The interfacial debonding is characterized by the diagram of interface (bond) shear stress $\tau$ versus relative stress displacement $v$ shown in Fig. $1(c)$, where $\tau_{s}=$ initial bond strength (initial cohesion), $\tau_{d}=$ residual bond stress at sliding interface, and $\nu_{0}=$ critical slip determining the slope of the $\tau(v)$ diagram, which is assumed to be linear. The fiber and matrix are elastic, characterized by Young's elastic moduli $E_{f}$ and $E_{m}$. Although in reality $\tau_{s}$ and $\tau_{d}$ are pressure-dependent, in a one-dimensional model they must be assumed to be constant. The interface shear stress at the softening portion is 


$$
\tau=\tau_{s}\left(1-\frac{v}{v_{0}}\right)
$$

The cross-hatched area in Fig. 1(c) represents the bond fracture energy, which is expressed as

$$
G_{f}=\frac{1}{2} \tau_{s} \tau_{b}\left(1-\frac{\tau_{d}}{\tau_{s}}\right)^{2}
$$

Let $z$ be the longitudinal coordinate. The fiber has a free end at $z=-l$. We will study two types of test: (1) Pull-pull, in which the cylinder representing the matrix has a free end at $z=0$ and is supported at the opposite end [Fig. 2(a)]; and (2) pull-push, in which the matrix cylinder is supported at $z=0$ [Fig. 2(b)]. First we consider the pull-pull test and leave consideration of the pull-push test to the end.

Equilibrium of a small element of the fiber, of length $\delta z$, requires that $\delta \sigma\left(\pi d^{2} / 4\right)=\tau(\pi d) \delta z$, which yields

$$
\frac{d \sigma}{d z}=\frac{4 \tau}{d}
$$

where $\sigma=$ normal stress in the fiber. Equilibrium in the cross sections of fiber and matrix requires that $\sigma A_{f}+\sigma_{m} A_{m}=\sigma_{a} A_{f}$, which yields

$$
\sigma_{m}=\frac{E_{m}}{E_{f}} \phi\left(\sigma_{a}-\sigma\right)
$$

where $\sigma_{a}=$ applied pull-out stress $\left(\sigma_{a}=P / A_{f}\right.$ where $P$ is the pullout load), $\sigma_{m}=$ normal stress in the matrix cylinder, $\phi=A_{f} E_{f} / A_{m} E_{m}, A_{f}=\pi d^{2}$, and $A_{m}=\pi\left(D^{2}-d^{2}\right)$.

Noting that the difference between the strains in the fiber and the matrix is $d v / d z$, we have $d v / d z=\sigma / E_{f}-\sigma_{m} / E_{m}$, which yields

$$
\frac{d v}{d z}=\frac{1+\phi}{E_{f}} \sigma-\frac{\phi}{E_{f}} \sigma_{a}
$$

The displacement at the end of embedment (Fig. 2), $z=0$, is

$$
\delta=v(-L)+\int_{-L}^{0} \frac{\sigma}{E_{f}} d z=\frac{v(0)+\phi v(-L)}{1+\phi}+\frac{\phi \sigma_{a} L}{(1+\phi) E_{f}}
$$

For the case of softening slip, the differential equation for the fiber stresses ensues by differentiating (5) and substituting (3) and (1)

$$
\frac{d^{2} \sigma}{d z^{2}}+\omega^{2} \sigma=\frac{\phi}{1+\phi} \omega^{2} \sigma_{a}
$$

in which

$$
\omega^{2}=\frac{4(1+\phi) \tau_{s}}{E_{f} v_{0} d}
$$

At the cross sections with no interface slip (no shear crack), the strains in the fiber and the matrix cylinder are equal, i.e., $\sigma / E_{f}=\sigma_{m} / E_{m}$; this yields 


$$
\sigma=\frac{\phi}{1+\phi} \sigma_{a}
$$

\section{ANALYSIS OF PULL-PULL TEST}

In the pull-pull case, two interface cracks grow from both ends of the fiber until they join. At that moment the maximum applied stress $\sigma_{a}=\sigma_{N}$, representing the nominal strength, is reached. If the load is controlled, failure occurs at that moment. The postpeak softening is observable only when the fiber displacement at the end is controlled, except when the response diagram exhibits a snapback. The snapback, as we will see, occurs for sufficiently large sizes.

Because of the discontinuities at the beginning of slip and at the attainment of residual bond shear strength, several stages must be distinguished in the solution. The number of states to consider is reduced in the case that $\phi=1$ (Fig. 3). Therefore we restrict attention to this case, although the general conclusions and implications for the size effect are the same for any $\phi$. For $\phi=1$, we have $\omega^{2}=8 \tau_{s} / E_{f} t_{0} d$. The stages we must distinguish are as follows:

1. The initial stage, in which there are two separate cracks emanating from the ends of the fiber and the shear stress is everywhere larger than the residual strength $\tau_{d}$,

2. The final stage, in which the two cracks have joined into one and the residual strength $\tau_{d}$ has been reached at both ends.

3. The intermediate stage, in which one must distinguish two cases: (1) The two cracks join before $\tau$ reaches $\tau_{d}$ at the ends; or (2) the shear stress $\tau_{d}$ is reached before the cracks join.

\section{Initial Stage}

In the middle portion of the fiber there is no slip and the shear stress $\tau$ $=0$. The maximum $\tau$ occurs at the fracture tips $z=-l_{i}$, at which $\tau=\tau_{s}$; $i=1,2$ refer to the right and left parts. From (7), for parts I and II

$$
\tau_{i}=\tau_{s} \cos \omega\left(z+l_{i}\right)
$$

From (1), the interface slip is

$$
v=v_{0}\left[1-\cos \omega\left(z+l_{i}\right)\right]
$$

The slip increases from the crack tip to the end of the crack. The distances $l_{i}$ of the tips from the right end of the fiber $(z=0)$ are $l_{1}=a$ and $l_{2}=L$

- $a$, where $a=$ length of each crack

$$
a=\frac{1}{\omega} \arcsin \left(\frac{\omega d}{8 \tau_{s}} \sigma_{a}\right)
$$

Between the cracks, the stress in the fiber is $\sigma=\sigma_{a} / 2$. From the crack tips to the crack ends, the stress in the fiber increases as

$$
\sigma_{t}=\frac{\sigma_{a}}{2}\left[1+\frac{\sin \omega\left(z+l_{i}\right)}{\sin \omega a}\right]
$$




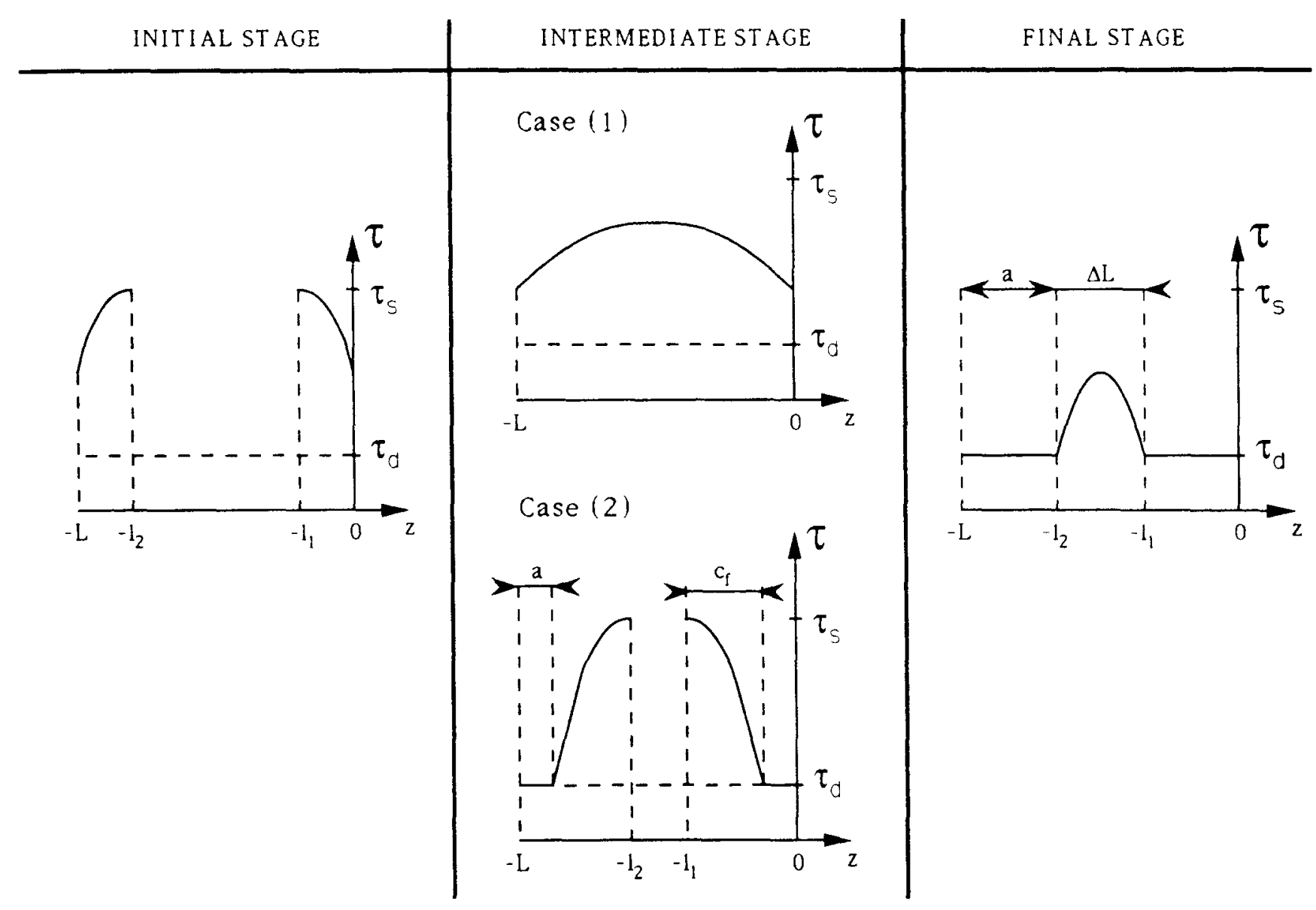

FIG. 3. Fracture Process Zones and Zones of Residual Stress along Fiber for Various Stages of Loading 
The displacement of the end increases with $\sigma_{a}$, and the diagram of $\sigma_{a}(\delta)$ is given, in the first stage, by

$$
\delta_{1}=\frac{L \sigma_{a}}{2 E_{f}}+\tau_{0}\left[1-\sqrt{1-\left(\frac{\omega \sigma_{a} d}{8 \tau_{s}}\right)^{2}}\right]
$$

The diagram of $\sigma_{a}$ versus $\delta$ has a negative curvature. The transition to the intermediate stage occurs when $\sigma_{a}$ reaches a critical value that is the smaller of the following two values:

$$
\sigma_{a}^{*}=\frac{8 \tau_{s}}{\omega d} \sin \frac{\omega L}{2} ; \quad \sigma_{a}^{* *}=\frac{8 \tau_{s}}{\omega d} \sqrt{1-\frac{\tau_{d}^{2}}{\tau_{s}^{2}}}
$$

The critical value is $\sigma_{a}^{*}$ if $\omega L$ is small enough and $\sigma_{a}^{* * *}$ if $\omega L$ is large enough.

\section{Intermediate Stage}

Case 1 of the intermediate stage, already defined [Fig. 3(b)], occurs if $\omega L<2 \arccos \left(\tau_{d} / \tau_{s}\right)$. Otherwise case 2 occurs.

In case 1 , the cracks have already joined and the interface shear stress is everywhere smaller than $\tau_{s}$, but slightly larger than $\tau_{d}$. According to (1)

$$
\tau=\sigma_{a} \frac{\omega d \cos \omega\left(z+\frac{L}{2}\right)}{8 \sin \omega \frac{L}{2}}
$$

The stress in the fiber (Fig. 2) increases from the left end ( $z=-L)$ to the right end $(z=0)$ and is

$$
\sigma=\frac{\sigma_{a}}{2}\left[1+\frac{\sin \omega\left(z+\frac{L}{2}\right)}{\sin \omega \frac{L}{2}}\right]
$$

The displacement $\delta$ of the end of the fiber is, for the first case of the intermediate stage

$$
\delta_{21}=v_{0}+\frac{L \sigma_{a}}{2 E_{f}}\left(1-\frac{2}{\omega L} \cot \frac{\omega L}{2}\right)
$$

The stress in the fiber varies from $\sigma_{a}^{*}$ to

$$
\sigma_{a}^{I}=\frac{8 \tau_{d}}{\omega d} \tan \frac{\omega L}{2}
$$

for which the residual interface shear stress $\tau_{d}$ is reached at the end. Because $\omega L<2 \arccos \left(\tau_{d} / \tau_{s}\right), \sigma_{a}^{l}$ is always smaller than $\sigma_{a}^{*}$, and so the failure occurs at $\sigma_{a}=\sigma_{N}=\sigma_{a}^{*}$. The equilibrium path of the structure exhibits snapback if $L$ is sufficiently large or $\tau_{d}$ is sufficiently small. Precisely, the condition of snapback is 


$$
2 x_{0}<\omega L<2 \arccos \frac{\tau_{d}}{\tau_{s}}
$$

in which $x_{0}=$ the root of $x_{0} \tan x_{0}=1$, i.e., $x_{0}=0.8603$.

In case 2 of the intermediate stage, i.e., for $\omega L>2 \arccos \left(\tau_{d} / \tau_{s}\right)$, there are two cracks [Fig. 3(b)]. The interface fracture process zone exhibits linear softening and its length is $c_{f}$. The fracture process zone of length $c_{d}$ is at constant residual interface shear stress $\tau=\tau_{d}$

$$
c_{f}=\frac{1}{\omega} \arccos \frac{\tau_{d}}{\tau_{s}} ; \quad c_{d}=\frac{d}{8 \tau_{d}}\left(\sigma_{a}-\sigma_{a}^{* *}\right)
$$

and $a=l_{1}=L-l_{2}=c_{f}+c_{d}$. With $G_{f}$ defined by (2) and $\omega$ by (8), the fracture process zone is $c_{f}=\kappa_{1} \sqrt{l_{0} d}$ in which $\kappa_{1}=(\pi / 4) \sqrt{E_{f} / E_{m}}$ and $l_{0}=$ $E_{m} G_{f} / \tau_{s}^{2}$ when $\tau_{d}=0$. In the general case, $\kappa_{1}$ depends on both the fiber proportion in the matrix and the elastic moduli

$$
\kappa_{1}=\frac{\pi}{2} \sqrt{\frac{E_{f}}{2(1+\phi) E_{m}}}
$$

The expression for the normal stress in the portion of the fiber that has a linearly varying interface shear stress is the same as for the initial stage. For the portion of the fiber that has a constant interface shear stress, the normal stress is linear in $z$ if $\tau_{d}=0$, and otherwise it is constant. The end displacement $\delta$ increases with $\sigma_{a}$, and the diagram of $\sigma_{a}$ versus $\delta$ has a positive curvature

$$
\delta_{22}=\frac{L \sigma_{a}}{2 E_{f}}+v_{0}\left(1-\frac{\tau_{d}}{\tau_{s}}\right)+\frac{d}{16 \tau_{d} E_{f}}\left(\sigma_{a}^{2}-\sigma_{a}^{* 2}\right)
$$

Failure occurs when $\sigma_{a}=\sigma_{N}=\sigma_{a}^{I I}$, which is always larger than $\sigma_{a}^{* *}$

$$
\sigma_{a}^{\mathrm{II}}=\sigma_{a}^{* *}+\frac{4 \tau_{d}}{\omega d}\left(\omega L-2 \arccos \frac{\tau_{d}}{\tau_{s}}\right)
$$

\section{Final Stage}

The softening zone is now localized in the middle of the specimen [Fig. $3(c)$ ]. Its length $\Delta L$ gradually decreases to 0 . The length of the fracture zone, in which $\tau=\tau_{d}$, is $c_{d}=(L-\Delta L) / 2$. For a given applied stress $\sigma_{a}$, $\Delta L$ is the solution of

$$
\sigma_{a}=\frac{4 \tau_{d}}{d}\left(L-\Delta L+\frac{2}{\omega} \tan \frac{\omega \Delta L}{2}\right)
$$

Displacement $\delta$ at the end of the fiber is

$$
\delta_{3}=\frac{L \sigma_{a}}{2 E_{f}}+v_{0}\left(1-\frac{\tau_{d}}{\tau_{s}}\right)+c_{d}\left(\frac{\sigma_{a}}{E_{f}}-\frac{4 c_{d} \tau_{d}}{E_{f} d}\right)
$$

Note that $d \sigma_{a} / d(\Delta L)$ is always positive, and $d(\Delta L)=-2 d c_{d}$ is always negative. Therefore, $d \sigma_{a}$ is negative, and the equilibrium path decreases 
with the applied stress. Therefore, failure occurs in this case before the final stage is reached.

The condition of snapback is

$$
\frac{\omega\left(L+2 c_{d}\right)}{2} \tan \frac{\omega \Delta L}{2}-1>0
$$

At $L / d=$ constant, $\omega L \propto \sqrt{d}$ when $0 \leq \omega \Delta L / 2 \leq \arccos \left(\tau_{d} / \tau_{s}\right)$, and so snapback must occur for sufficiently large sizes.

\section{SIZE EFFECT}

The scaling law is the most important attribute of any physical theory. In the classical theories of elasticity or plasticity, the problem of scaling law has not received much attention, because the law is very simple - the nominal strength is independent of structure size. In the mechanics of damage and nonlinear fracture mechanics, the problem of scaling or size effect has received major attention in recent years, principally because there is a strong effect of size on the nominal strength and the scaling law is more complex, representing a transition from elasticity (or plasticity) to linear elastic fracture mechanics, in which the nominal strength is inversely proportional to the square root of the structure size (Bažant 1984, 1992; Bažant and Cedolin 1991).

The size effect can be defined only for structures with similar geometries and similar cracks. Therefore, we consider the ratios $D / d$ and $L / d$ to be constant and choose the fiber diameter $d$ to play the role of characteristic dimension of the structure. We note that, in this case, $\omega d$ and $\omega L$ are both proportional to $\sqrt{d}$. The applied pullout stress $\sigma_{a}$ at maximum load may be employed as the nominal strength $\sigma_{N}$. The value of $\sigma_{N}$ can be calculated from (15) if $\omega L<2 \arccos \tau_{d} / \tau_{s}$, and otherwise from (24).

For numerical examples, we consider the material properties $\tau_{s}=31$ $\mathrm{MPa}, \tau_{d}=3 \mathrm{MPa}, v_{0}=0.021 \mathrm{~mm}$, and $E_{f}=200 \mathrm{GPa}$, and run the calculations for sizes $d=1,2.9,6.4$, and $12.7 \mathrm{~mm}$ at constant ratio $L / d=$ 4. The results are plotted in Fig. 4. It is apparent that the maximum pullout stress decreases with increasing size. Furthermore, the type of the loaddisplacement diagram changes; for the smallest size we have a gradual postpeak softening, for the next size we have a nearly vertical stress drop, and for the largest two sizes we have snapback instability right after the peak. This behavior is typical of the size effect in all structures exhibiting damage localization or nonlinear fracture. The size effect is caused by increasing localization of the softening regions along the fiber length as $d$ increases. The softening region at maximum load, which represents the fracture process zone and is characterized by stress values between $\tau_{s}$ and $\tau_{d}$ (Fig. 1), extends in small specimens over a large portion of the fiber length and in large specimens over a small portion of the fiber length. This behavior is similar to all other failures due to damage growth or nonlinear fracture.

The size effect obtained for our example is shown in Fig. 5 by the diagram of $\log \left(\sigma_{N}-\sigma_{0}\right)$ versus $\log d$, where $\sigma_{0}=$ the residual fiber strength corresponding to the residual interface bond stress $\tau_{d}$.

Let us now examine the asymptotic behavior. In the limit of small sizes, $d \rightarrow 0$, we obtain

$$
\sigma_{N}=\sigma_{a}^{*}=4 \tau_{s} \frac{L}{d}=\text { constant }
$$



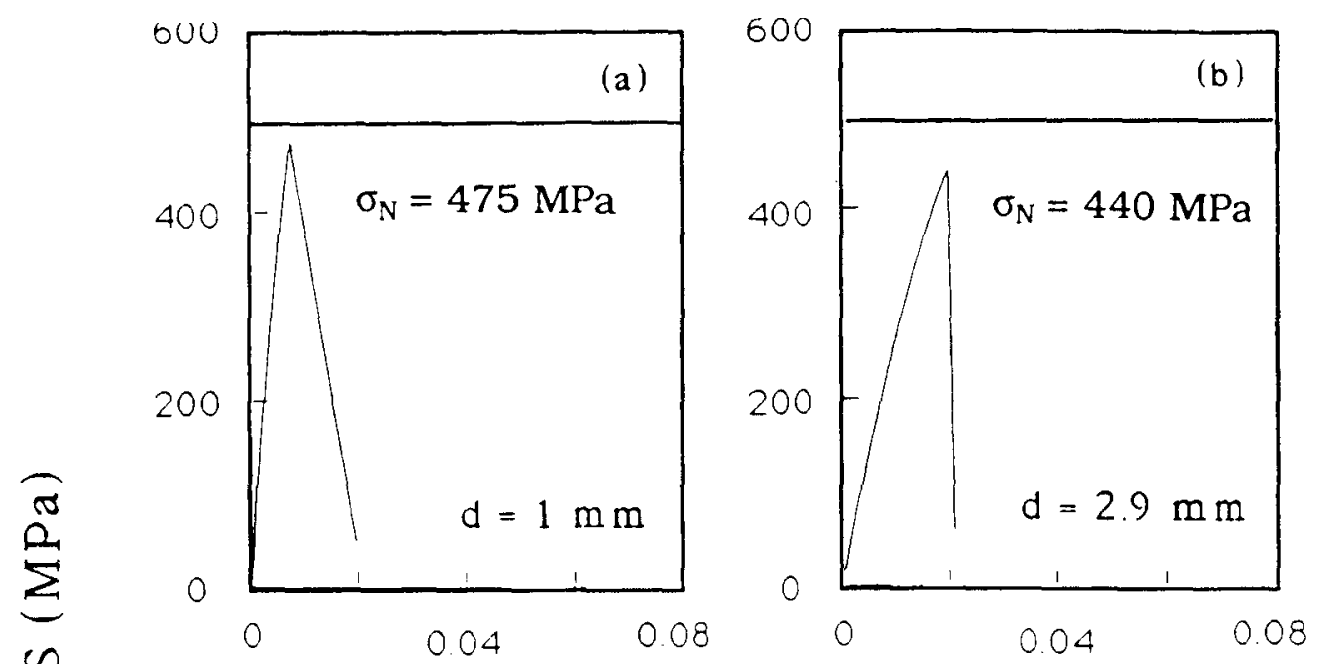

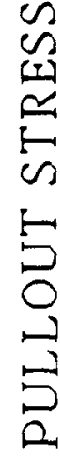
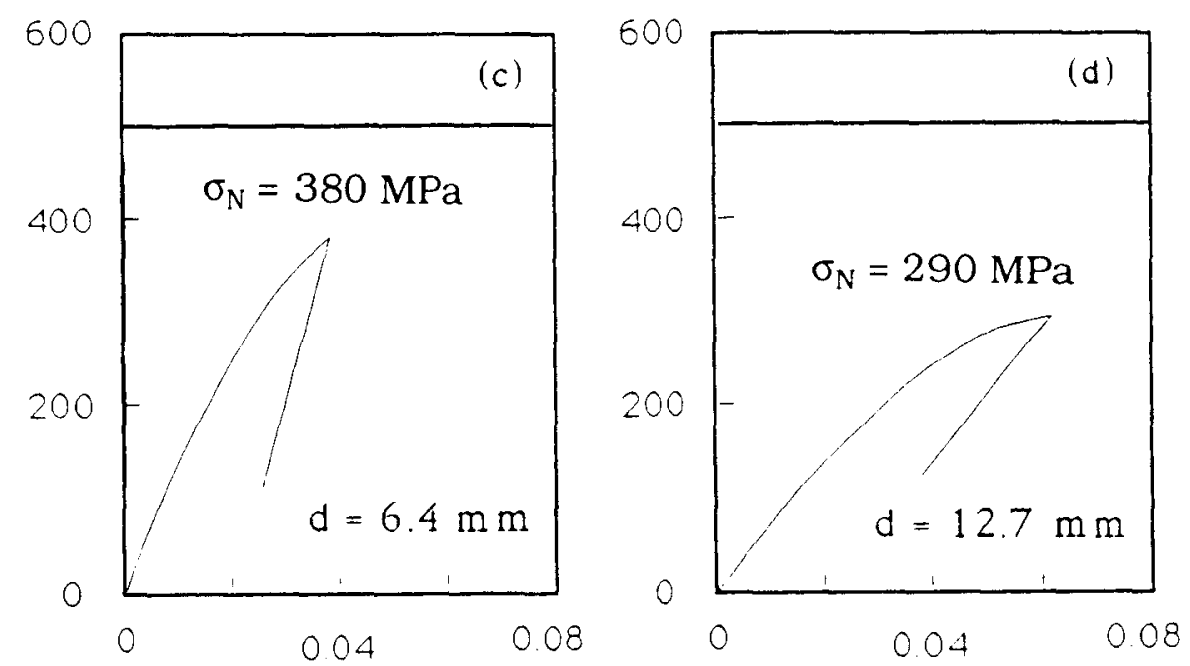

\section{DISPLACEMENT ( $\mathrm{m} \mathrm{m})$}

FIG. 4. Diagrams of Pullout Stress versus Displacement for Tests of Similar Geometry and Different Sizes

In the limit of the large sizes, $d \rightarrow \infty$, we obtain

$$
\sigma_{N}=\sigma_{a}^{\prime \prime}=\sigma_{0}+\frac{\sqrt{8 \tau_{s} E_{f} \tau_{0}^{\prime}}}{\sqrt{d}}\left(\sqrt{1-\frac{\tau_{d}^{2}}{\tau_{s}^{2}}}-\frac{\tau_{d}}{\tau_{s}} \arccos \frac{\tau_{d}}{\tau_{s}}\right)
$$

in which $\sigma_{0}=$ the residual pullout stress of the fiber when the interface is completely debonded and softened to $\tau_{d}$

$$
\sigma_{0}=4 \tau_{d} \frac{L}{d}
$$

According to (29), the basic form of the size effect for the large sizes is

$$
\sigma_{N}-\sigma_{0} \propto \frac{1}{\sqrt{d}}
$$




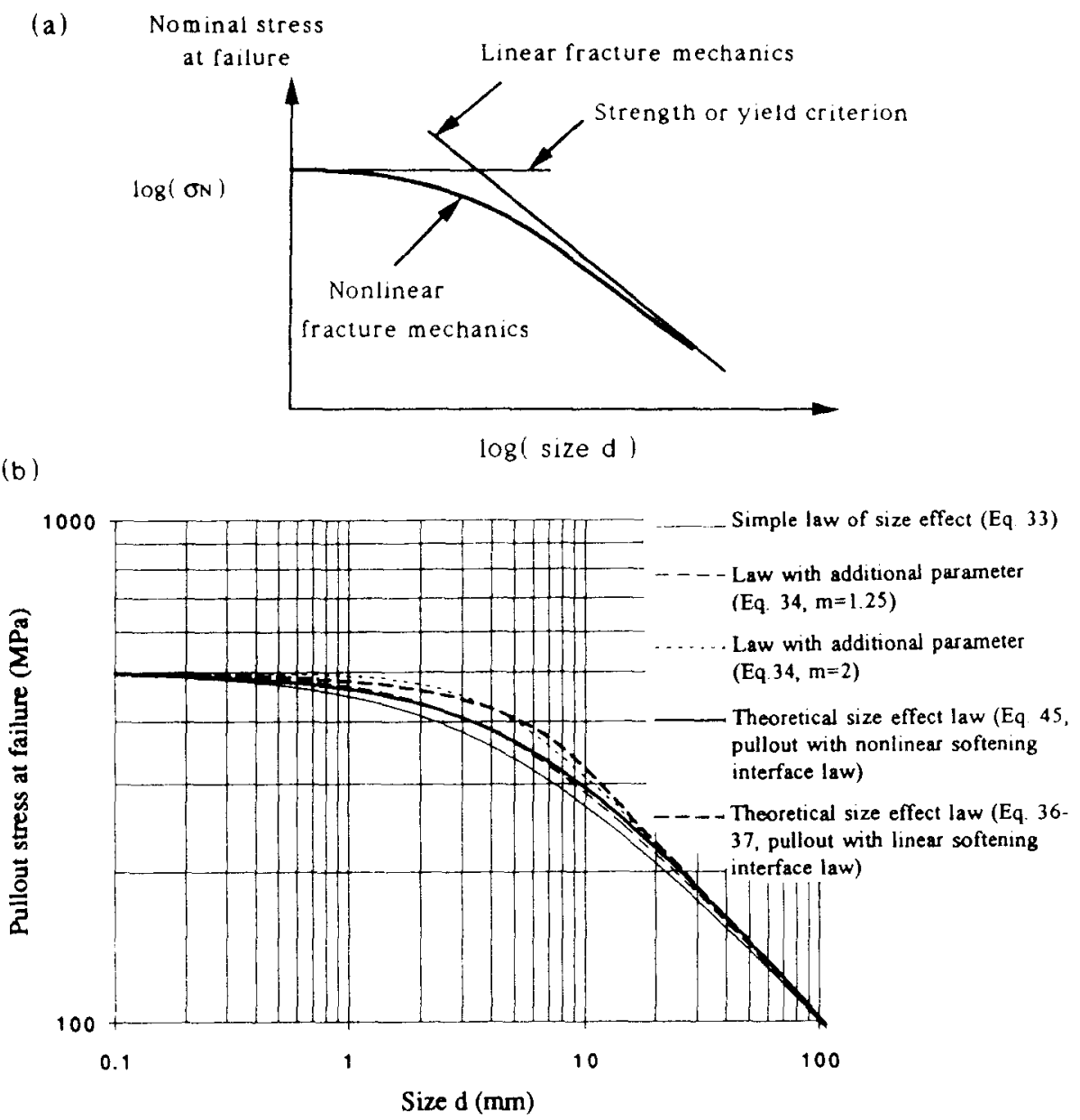

FIG. 5. (a) Size Effect Law Proposed by Bažant (1984); and (b) Comparison of Calculated Size Effect to General Forms of Size Effect Law for Quasibrittle Fracture

Except for the presence of $\sigma_{0}$, this represents the size effect characteristic of linear elastic fracture mechanics. In the plot of Fig. 5, it corresponds to the inclined straight-line asymptote of slope $-1 / 2$.

The size effect obtained by the present analysis and shown in Fig. 5 agrees with the general size effect of damage mechanics or nonlinear fracture. Under the hypothesis that the energy dissipated at failure is a smooth function of both the specimen (or structure) size and the fracture process zone size, with the latter being a material property, it was shown (Bažant 1985) by dimensional analysis and similitude arguments that, in general

$$
\sigma_{N}=B f_{t}^{\prime}\left[\xi\left(1+\xi^{-1}+A_{1} \xi^{2}+A_{2} \xi^{3}+\cdots\right)\right]^{-1 / 2 m} ; \quad \xi=\left(d / d_{0}\right)^{m}
$$

Here $f_{t}^{\prime}=$ the tensile strength of the material, introduced strictly for convenience, and $m, B, d_{0}, A_{1}, A_{2}, \ldots$ are positive empirical coefficients. Eq. (32) represents an asymptotic series expansion with respect to an infinitely large specimen. It was further shown (Bažant 1987) that for size ranges up to about 1:20, the asymptotic series can be truncated after the linear term and that, for most applications to concrete and rock, one can take $m=1$. Thus (32) reduces to the size effect law (Bažant 1984) 


$$
\sigma_{N}=\frac{B f_{t}^{\prime}}{\sqrt{1+\beta}} ; \quad \beta=\frac{d}{d_{0}}
$$

For materials with a residual strength, represented here by $\tau_{d}, \sigma_{N}$ must be replaced in the foregoing equations with $\sigma_{N}-\sigma_{0}$ where $\sigma_{0}$ is the residual nominal strength. Thus, truncation of (32) after the linear term yields the law

$$
\sigma_{N}-\sigma_{0}=B f_{t}^{\prime}\left(1+\beta^{m}\right)^{-1 / 2 m}
$$

and the simple size effect law $(m=1)$ in (33) is generalized as

$$
\sigma_{N}-\sigma_{0}=\frac{B f_{i}^{\prime}}{\sqrt{1+\beta}}
$$

It is obvious that, for $d \gg d_{0},(33)-(35)$ reduce to $\sigma_{N}-\sigma_{0} \propto d^{-1 / 2}$, which is the form of size effect exhibited by every formula of linear elastic fracture mechanics. For $d<<d_{0}$, (33) or (35) reduces to $\sigma_{N}=$ constant (no size effect), which is characteristic of elasticity or plasticity. For the intermediate values of size $d,(33)$ or (35) describes a gradual transition between these two asymptotic cases.

Matching the asymptotes to those calculated for fiber pullout, the simple size effect law in (33) gives in Fig. 5 the plot shown by the solid curve $\left(B f_{t}^{\prime}=500 \mathrm{MPa}, d_{0}=4.2 \mathrm{~mm}\right)$.

The presently calculated size effect law may be rewritten for $\sigma_{0}=\tau_{d}=$ 0 as follows:

$$
\begin{gathered}
\sigma_{N}=\frac{B f_{t}^{\prime}}{\sqrt{\beta}} \sin \sqrt{\beta} \quad \text { if } \quad \beta \leq \frac{\pi^{2}}{4} \\
\sigma_{N}=\frac{B f_{t}^{\prime}}{\sqrt{\beta}} \quad \text { if } \quad \beta>\frac{\pi^{2}}{4}
\end{gathered}
$$

These results are exact for the pull-push problem for any $\phi$, as we will see in the next section.

Eq. (35) is not identical to (36)-(37), but it can be made nearly identical for a certain value of $m$. This value can be estimated by requiring (34) and (36) to coincide for $d=d_{0}$ or $\beta=1$. This yields

$$
m=-\frac{\ln 2}{2 \ln (\sin 1)}=2.009 \approx 2
$$

For $m=2$, the agreement of (36) and (37) with (35) becomes virtually perfect.

For tensile fracture, the value of exponent $m$ is known to be related to the shape of the strain-softening diagram (Bažant 1985). Striving for the simplest analytical solution possible, we have assumed this diagram to be linear [Fig. 1(c)]. For tensile fracture, it was shown that a softening diagram with a progressively decreasing slope and a long tail yields a more gradual transition in the size effect plot. It may be expected that if Fig. 1(c) were replaced by such a softening diagram, the calculated size effect could be made to match the dashed curve in Fig. 5, corresponding to the simple size effect law in (35). It remains to be seen whether the actual behavior of interfaces corresponds to the simple case $m=1$ (as it approximately does for tensile fracture of concrete), or an $m$-value very different from 1 needs 
to be used. To illuminate this question, the simple nonlinear softening law $\tau=\tau_{s} \exp (-b v)[$ Fig. $1(d)]$ will be considered next.

\section{SIZE EFFECT LAW FOR NONLINEAR SOFTENING INTERFACE BEHAVIOR}

The pullout equations (3) and (5), complemented by the general nonlinear law $\tau=\tau(v)$, lead to the general nonlinear differential equation for $v(z)$

$$
\frac{d^{2} v}{d z^{2}}=k \tau(v), \quad \text { with } \quad k=\frac{4(1+\phi)}{E_{f} d}
$$

which is valid for both the pull-pull and pull-push tests. The axial stress in the fiber is given by $\sigma(z)=E_{f}(1+\phi)^{-1} v^{\prime}(z)+\phi(1+\phi)^{-1} \sigma_{a}$ for the pull-pull test and by $\sigma(z)=E_{f}(1+\phi)^{-1} v^{\prime}(z)$ for the pull-push test. The boundary conditions are $v^{\prime}=0$ at the tip of interface crack $(v=0), \sigma=$ $\sigma_{a}$ at $z=0$, and $\sigma=0$ at $z=-L$. For simplicity, as before, the pull-pull case will be studied for $\phi=1$, and the pull-push case for any $\phi$.

The general solution of (39) is

$$
\int \frac{d v}{\sqrt{\int \tau(v) d v}}=(\sqrt{2 k}) z
$$

To make integration easy, we will consider

$$
\tau(v)=\tau_{s} \exp (-b v)
$$

The residual shear stress $\tau_{d}$ is here taken equal to zero, and $b$ is related to the fracture energy $G_{f}$ by $b=\tau_{s} / G_{f}$. From (40)

$$
v=\frac{2}{b} \ln \left\{\cosh \left[\sqrt{2 k G_{f}} \frac{b\left(z+l_{i}\right)}{2}\right]\right\}
$$

The axial stress for the pull-pull case $(\phi=1)$ is

$$
\sigma=\frac{E_{f} \sqrt{2 k G_{f}}}{2} \tanh \left[\sqrt{2 k G_{f}} \frac{b\left(z+l_{i}\right)}{2}\right]+\frac{\sigma_{a}}{2}
$$

and for the pull-push case

$$
\boldsymbol{\sigma}=\frac{E_{f} \sqrt{2 k G_{f}}}{1+\phi} \tanh \left[\sqrt{2 k G_{f}} \frac{b(z+l)}{2}\right]
$$

The stress at failure is reached when the interface is debonded along all of its length.

The size-effect law for pullout with exponential softening and no residual stress may now be written as $\left(\beta=d / d_{0}\right)$

$$
\sigma_{N}=\frac{B f_{t}^{\prime}}{\sqrt[r]{\beta}} \tanh \sqrt{\beta}
$$

where $B f_{t}=4 \tau_{s} L / d, d_{0}=16 E_{f} G_{f} /\left(B f_{t}^{\prime}\right)^{2}$ for the pull-pull test with $\phi=1$, and $d_{0}=8 E_{f} G_{f}(1+\phi)^{-1} /\left(B f_{t}^{\prime}\right)^{2}$ for the pull-push test.

Again, to match (34) closely to (45), we require them to coincide for $\beta$ $=1\left(d=d_{0}\right)$. This condition yields 


$$
m=-\frac{\ln 2}{2 \ln (\tanh 1)} \simeq 1.25
$$

As might have been expected, $m$ is now found to be much closer to 1 than for the linear softening. This confirms the dependence of $m$ on the shape of the interface stress-slip law. The corresponding size effect curves are plotted in Fig. 5(b). We see the theoretical curve agrees almost perfectly with the size effect law with additional parameter (34) and is quite close to the simple size effect law (35).

The foregoing analysis with a softening exponential stress-slip law does not take in account the residual strength $\sigma_{0}$ of the interface. For its effect one must refer to our solution for linear softening.

The general conclusion of our nonlinear analysis is that the influence of the shape of the shear stress-slip curve on the size effect is appreciable only for the transitional sizes. For a softening stress-slip law of declining slope, the size effect is closer to the simple formula (33) than for a stress-slip linear law. The asymptotes of the size effect curve in a log-log plot remain the same; the interface strength $\tau_{s}$ governs the failure for very small sizes, and the interface fracture energy $G_{f}$ the failure for very large sizes.

According to (40), closed-form analytical solutions could be obtained also for stress-slip laws other than (1) or (41).

\section{IDENTIFICATION OF INTERFACE PROPERTIES FROM SIZE EFFECT MEASURED IN PUSH-PULL TESTS}

In the mechanics of tensile fracture, the measured size effect can be exploited to determine the material fracture characteristics (Bažant 1987; Bažant and Pfeiffer 1987; Bažant and Kazemi 1990). The same must be possible for fiber pullout.

Indeed, after calculating the asymptotes of the size effect plot, the size effect parameters for linear softening can be identified by matching these asymptote with (28) and (29). This yields

$$
\begin{gathered}
B f_{t}^{\prime}=4\left(\tau_{s}-\tau_{d}\right) \frac{L}{d} \\
d_{0}=\frac{8 \tau_{s} E_{f} \prime_{0}}{\left(B f_{t}^{\prime}\right)^{2}}\left(\sqrt{1-\frac{\tau_{d}^{2}}{\tau_{s}^{2}}}-\frac{\tau_{d}}{\tau_{s}} \arccos \frac{\tau_{d}}{\tau_{s}}\right)^{2}
\end{gathered}
$$

When the size effect law is to be matched to experimental data on $\sigma_{N}$, parameters of (33) can be easily identified by linear regression $Y=A X+$ $C$, where $X=d, Y=1 /\left(\sigma_{N}-\sigma_{0}\right)^{2}, B f_{1}^{\prime}=1 / \sqrt{C}$, and $d_{0}=C / A$. A similar linear regression is possible for (34).

As an example, we will use the test data of Bažant and Sener (1988) (the circled points in Fig. 5). These data are for pullout of reinforcing bars from concrete cubes. We use these data only to illustrate the procedure while being fully aware that the failure mode observed in these tests did not fit the assumptions of the present analysis. The failure started by radial splitting cracks emanating from the bar. These cracks, which were caused mainly by lugs on the reinforcing bars, cannot be described by a one-dimensional model. Had smooth rather than deformed bars been used, the failure would have been due only to interface slip, and then the present example would represent the reality rather than just a mere illustration of the procedure.

Deformed reinforcing bars of yield strength $414 \mathrm{MPa}$ and diameters 2.9, 
6.4 , and $12.7 \mathrm{~mm}$ were used. In each cube, there was one bar parallel to one edge of the cube and sticking out at the center of one face. The embedment length of the bar was $L=4 d$. The size effect law parameters, identified previously (Bažant and Sener 1988) were $B f_{t}^{\prime}=500 \mathrm{MPa}, d_{0}=2.1 \mathrm{~mm}$, and $\sigma_{0}=0$.

For the purpose of analyzing these data, the solution for the pull-push test has also been derived

$$
\text { for } \omega L \leq \arccos \frac{\tau_{d}}{\tau_{s}}: \quad \sigma_{N}=\frac{4 \tau_{s}}{\omega d} \sin \omega L
$$

for $\omega L>\arccos \frac{\tau_{d}}{\tau_{s}}: \quad \sigma_{N}=\sigma_{0}+\frac{4 \tau_{s}}{\omega d}\left(\sqrt{1-\frac{\tau_{d}^{2}}{\tau_{s}^{2}}}-\frac{\tau_{d}}{\tau_{s}} \arccos \frac{\tau_{d}}{\tau_{s}}\right)$

in which $\omega$ is given by $(8)$ and $\sigma_{0}=4 \tau_{d} L / d, \phi=A_{f} E_{f} / A_{m} E_{m}$. Knowing the exponent $m$, which is here taken as $m=1$ [same as Bažant and Sener (1988)], we can use the aforementioned linear regression plot $Y=A X$

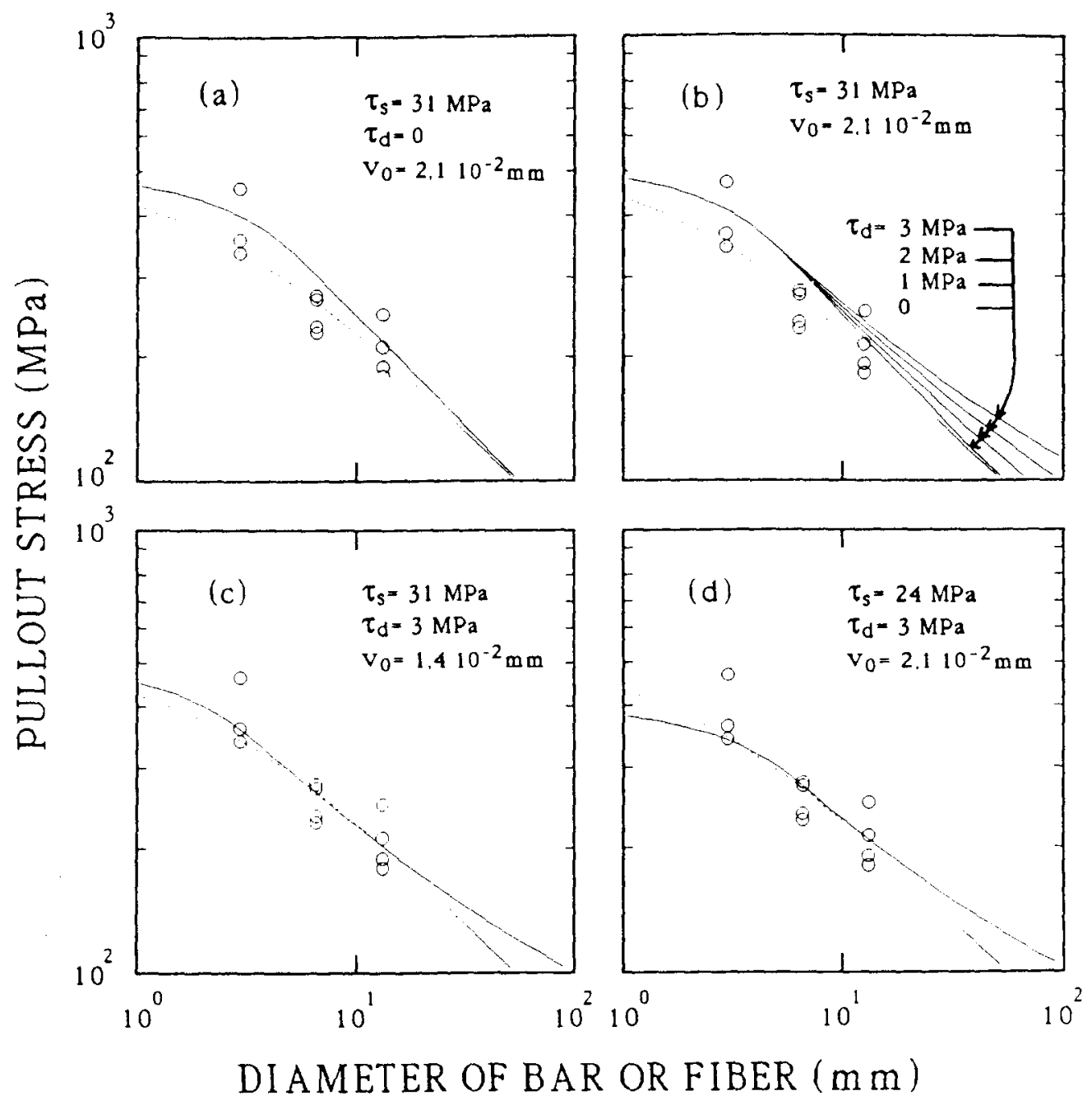

FIG. 6. Test Data for Bar Pullout (Circular Points), Used as Illustrative Example, and Comparisons with Present Solution (Solid Curves) and with Simple Form of Size Effect Law (Dashed Curve) 
$+C$ to determine the size effect law parameters $B f_{1}^{\prime}$ and $d_{0}$. Matching the asymptotes, we get the following expressions for the interface properties:

$$
\begin{gathered}
\tau_{s}=\frac{d}{4 L} B f_{t}^{\prime}+\tau_{d} \\
v_{0}=\frac{1+\phi\left(B f_{i}^{\prime}\right)^{2}}{\tau_{s} E_{f}} d_{1}\left(\sqrt{1-\frac{\tau_{d}^{2}}{\tau_{s}^{2}}}-\frac{\tau_{d}}{\tau_{s}} \arccos \frac{\tau_{d}}{\tau_{s}}\right)^{2}
\end{gathered}
$$

Then, using the size-effect law parameters obtained by Bažant and Sener (1988), we get from (51) and (52) the following interface properties:

$$
\tau_{s}=31 \mathrm{MPa} ; \quad v_{0}=2.110^{-2} \mathrm{~mm} ; \quad G_{f}=325 \mathrm{~J} / \mathrm{m}^{2} \quad(53 a, b, c)
$$

The value of $\tau_{d}$ has been neglected in these calculations. The optimum fit by the size effect law given by (33) is shown by the dashed curve in Fig. $6(a)$, and the fit based on (49) and (50) with the values in (53) is given by the solid curve. Assuming progressively increasing values $\tau_{d}=0,1,2$, or $3 \mathrm{MPa}$, one obtains from (49) and (50) the solid curves shown in Fig. 6(b)$(d)$. Unfortunately, the scatter of the data is insufficient to decide which of these curves is more correct. To avoid such ambiguity and obtain better estimates of interface properties, tests of a broader size range (1:10) would be necessary. The required breadth of range is generally proportional to the coefficient variation of the statistical scatter.

It is planned to carry out size effect tests of pullout in which the failure occurs by slip alone (without radial cracks). Then it will be possible to give an example that is more than just an illustration of the procedure.

\section{CONCLUSIONS}

1. The one-dimensional simplification of the fiber (or bar) pullout problems allows a simple analytical solution yielding closed form expressions for the stress-displacement diagram as well as the size effect.

2. The solution shows that, for geometrically similar situations: (1) The maximum pullout stress decreases with increasing size (characterized for example by the fiber diameter); (2) the postpeak slope of the load-deflection diagram becomes steeper as the size increases; and (3) for a sufficiently large size, snapback failure is obtained.

3. An inevitable consequence of softening in the relation of interfacial shear stress versus slip displacement is localization of the fracture process zone along the interface, with a gradual approach to interface shear fracture. Due to localization, the distribution of the interface shear stress along the fiber or bar becomes strongly nonuniform, and the nonuniformity gets stronger as the size increases. The localization is the cause of size effect.

4. The solution confirms that the size effect is transitional between the case of elasticity or plasticity, for which there is no size effect, and the case of linear elastic fracture mechanics, for which the difference of the interface strength and the residual stress is inversely proportional to the square root of the size. This transitional size effect can be described by the approximate size effect law proposed by Bažant (1984) or its subsequent generalization with parameter $m$ controlling the shape of the size effect curve.

5. The transitional size effect is shown to depend on the shape of the 
interface stress-slip law. A declining slope of the stress-slip law leads to a more gradual and more extended transition in the size-effect plot.

6. Measurements of the size effect in fiber pullout can be exploited for determining the interface properties.

\section{ACKNOWLEDGMENTS}

Financial support under AFOSR Grant 91-0140 to Northwestern University (monitored by Jim Chang) is gratefully acknowledged. The general size effect studies were partially supported by Center for Advanced CementBased Materials at Northwestern University. The second writer, Rodrigue Desmorat, acknowledges the friendly hospitality of the civil engineering department while he was a visiting research assistant at Northwestern University.

\section{APPENDIX. REFERENCES}

Bažant, Z. P. (1984). "Size effect in blunt fracture: concrete, rock, metal." J. Engrg. Mech., ASCE, 110(4), 518-535.

Bažant, Z. P. (1985). "Comment on Hillerborg's size effect law and fictitious crack model." Dei Poli anniversary volume, L. Cedolin et al., eds., Politecnico di Milano, Italy, 335-338.

Bažant, Z. P. (1992). "Scaling laws in mechanics of failure." Rep. No. 92-9/457s, Dept of Civ. Engrg., Northwestern Univ., Evanston, Ill.; also J. of Engrg. Mech., ASCE, $119(9), 1828-1866$.

Bažant, Z. P. (1985). "Fracture mechanics and strain-softening of concrete." Preprints, Seminar on finite element analysis of reinforced concrete structures, Japan Society for the Promotion of Science and U.S. National Science Foundation, Tokyo, Japan, 1, May, 47-69.

Bažant, Z. P. (1987). "Fracture energy of heterogeneous materials and similitude." SEM-RILEM Int. Conf. on Fracture of Concrete and Rock, Houston, Tex., S. P. Shah and S. E. Swartz, eds., June, 390-402.

Bažant, Z. P., and Cedolin, L. (1991). Stability of structures: elastic, inelastic, fracture and damage theories. Oxford University Press, New York, N.Y.

Bažant, Z. P., and Gambarova, P. G. (1980). "Rough cracks in reinforced concrete." J. Struct. Div., ASCE, 106(4), 819-842.

Bažant, Z. P., and Kazemi, M. T. (1990). "Determination of fracture energy, process zone length and brittleness number from size effect, with application to rock and concrete." Int. J. Fract., 44, 111-131.

Bažant, Z. P., and Pfeiffer, P. A. (1987). "Determination of fracture energy from size effect and brittleness number." ACI Mater. J., 84, 463-480.

Bažant, Z. P., and Sener, S. (1988). "Size effect in pullout tests." ACI Mater. J., $85,347-351$.

Beaumond, P. W. R., and Aleszka, J. C. (1978). "Cracking and toughening of concrete and polymer-concrete dispersed with short steel wires." J. Mater. Sci., $13,1749-60$.

Bowling, J., and Groves, G. W. (1979). "The debonding and pull-out of ductile wires from a brittle matrix." J. Mater. Sci., 14, 431-42.

Divakar, M. P., Fafitis, A., and Shah, S. P. (1987). "Constitutive model for shear transfer in cracked concrete." J. Struct. Div., ASCE, 113(5), 1046-1062.

Feenstra, P. H., de Borst, R., and Rots, J. G. (1991). "Numerical study on crack dilatancy." J. Engrg. Mech., ASCE, 117(4), 733-769.

Freund, L. B. (1992). "The axial force needed to slide a circular fiber along a hole in an elastic material and implications for fiber pull-out." European J. Mech., A/ Solids, 11(1), 1-19.

Fuller Jr., E. R., Butler, E. P., and Carter, W. C. (1991). "Determination of fibermatrix interfacial properties of importance to ceramic composite toughening." Proc., NATO Advanced Res. Workshop on Toughening Mechanisms in Quasi- 
Brittle Mat., S. P. Shah, ed., Kluwer Academic Publishers, Dordrecht, Netherlands, 385-404.

Gao, B. Y.-C., Mai, Y.-W., and Cotterell, B. (1988). "Fracture of fiber-reinforced materials." ZAMP, 39, 550-572.

Gray, R. J. (1984a). "Analysis of the effect of embedded fiber length on fiber debonding and pullout from an elastic matrix. Part 1: Review of theories." $J$. Mater. Sci., 19, 861-70.

Gray, R. J. (1984b). "Analysis of the effect of embedded fiber length on fiber debonding and pullout from an elastic matrix. Part 2: Review of theories." $J$. Mater. Sci., 19, 1680-91.

Hutchinson, J. W., and Jensen, H. M. (1990). "Models of fiber debonding and pullout in brittle composites with friction." Mech. Mater., 9, 139-63.

Lawrence, P. J. (1972). "Some theoretical considerations of fiber pull-out from an elastic matrix." J. Mater. Sci., 7, 1-6.

Leung, C. K. Y., and Li, V.C. (1990a). "A new theory for the debonding of discontinuous fibers in an elastic matrix." Mat. Res. Soc. Symp. Proc., 170, 4553.

Leung, C. K. Y., and Li, V.-C. (1990b). "Applications of a two-way debonding theory to short fibre composites." Composites, 21(4), 305-317.

Li, Z., Mobasher, B., and Shah, S. P. (1991). "Characterization of interfacial properties in fiber-reinforced cementitious composites." J. Am. Ceram. Soc., 74(9), $2156-64$.

Shah, S. P., and Ouyang, C. (1991). "Mechanical behavior of fiber-reinforced cement-based composites." J. Am. Ceram. Soc., 74(11), 2727-38, 2947-53.

Stang, H., Li, Z., and Shah, S. P. (1990). "Pullout problem: stress versus fracture mechanical approach." J. Eng. Mech., ASCE, 116(10), 2136-50.

Steif, P. S., and Hoysan, S. F. (1986). "On load transfer between imperfectly bonded constituents." Mech. Mater., 5, 375-382.

Wang, Y., Li, V.-C., and Backer, S. (1988). "Modeling of fibre pull-out from a cement matrix." Int. J. Cem. Compos. Lightweight Concr., 10(3), 143-149. 